\title{
Chemically resistant and thermally stable quantum dots prepared by shell encapsulation with cross-linkable block copolymer ligands
}

\author{
Jaewan Ko ${ }^{1}$, Byeong Guk Jeong $\mathbb{1}^{2}$, Jun Hyuk Chang ${ }^{3}$, Joonyoung F. Joung ${ }^{4}$, Suk-Young Yoon ${ }^{5}$, Doh C. Lee ${ }^{2}$,
} Sungnam Park ${ }^{4}$, June Huh', Heesun Yang ${ }^{5}$, Wan Ki Bae ${ }^{6}$, Se Gyu Jang ${ }^{7}$ and Joona Bang (1] ${ }^{1}$

\begin{abstract}
Endowing quantum dots (QDs) with robustness and durability have been one of the most important issues in this field, since the major limitations of QDs in practical applications are their thermal and oxidative instabilities. In this work, we propose a facile and effective passivation method to enhance the photochemical stability of QDs using polymeric double shell structures from thiol-terminated poly(methyl methacrylate- $b$-glycidyl methacrylate) (P(MMA- $b$ GMA)-SH) block copolymer ligands. To generate a densely cross-linked network, the cross-linking reaction of GMA epoxides in the PGMA block was conducted using a Lewis acid catalyst under an ambient environment to avoid affecting the photophysical properties of the pristine QDs. This provides QDs encapsulated with robust double layers consisting of highly transparent PMMA outer-shell and oxidation-protective cross-linked inner shell. Consequently, the resulting QDs exhibited exceptional tolerance to heat and oxidants when dispersed in organic solvents or QDnanocomposite films, as demonstrated under various harsh conditions with respect to temperature and oxidant species. The present approach not only provides simple yet effective chemical means to enhance the thermochemical stability of QDs, but also offers a promising platform for the hybridization of QDs with polymeric materials for developing robust light-emitting or light-harvesting devices.
\end{abstract}

\section{Introduction}

A myriad of academic research and industrial applications related to semiconductor nanocrystals, also referred to as quantum dots (QDs), has been conducted to take advantage of their outstanding color purity and unique size-dependent color-tunability due to the "quantum confinement effect"1-3. In addition to the above characteristics of QDs, their superb photoluminescence quantum yield (PLQY) and wide tunability over the entire visible range allow QDs to be potential key materials for displays $^{4-9}$, bioimaging ${ }^{10,11}$, photodetectors ${ }^{12,13}$, and

\footnotetext{
Correspondence: Wan Ki Bae (wkbae@skku.edu) or Se Gyu Jang (segyu.jang@kist.re.kr) or Joona Bang (joona@korea.ac.kr)

${ }^{1}$ Department of Chemical and Biological Engineering, Korea University, Seoul 02841, Republic of Korea

2Department of Chemical and Biomolecular Engineering, Korea Advanced Institute of Science and Technology (KAIST), Daejeon 34141, Republic of Korea Full list of author information is available at the end of the article.
}

photovoltaic cells ${ }^{14}$. Despite these advantages, QDs are extremely vulnerable to external stimuli such as heat ${ }^{15-17}$, oxidation $^{18-20}$, and moisture ${ }^{21}$, as it is well known that these factors create charge trap sites on the surface of QDs. For this reason, industrial and practical applications of QDs involving baking or drying processes that cause irreversible thermal and oxidation damage to QDs have been limited.

However, the most effective way to overcome the instabilities of QDs against external harsh environments is the passivation of emissive QDs with chemically robust, thick inorganic shells ${ }^{9,22-24}$. However, such core/shell heterostructuring encounters inherent limitations; building a thick shell beyond the critical thickness results in the creation of misfit defects near the core/shell interface that bring about a decrease in the PLQY of QDs ${ }^{25,26}$. The growth of thin oxide inorganic materials, such as 
$\mathrm{SiO}_{2}{ }^{22,27-29}$ or $\mathrm{Al}_{2} \mathrm{O}_{3}{ }^{30}$, was also devised to protect QDs from oxidation, but the growth of these shell materials is often accompanied by unexpected oxidation of QDs that yields irreversible degradation of QDs ${ }^{31-33}$.

Stabilizing QDs with ligands that strongly bind onto the QD surface has attracted tremendous attention as an alternative chemical means to endow QDs with photochemical or thermal stability ${ }^{34-37}$. Despite the decent progress made with thiolated ligands, the thermal dissociation of the ligands due to the relatively weak binding energy between ligands and inorganic surfaces ${ }^{38}$ has limited the use of ligand protective layers in application fields where QDs are exposed to heat. Recently, the crosslinked network formation of ligands was used to structurally inhibit the detachment of ligands from the surface of metal nanoparticles ${ }^{39-42}$, but this approach has not been directly applicable to QDs because it involves rather harsh chemical reaction conditions for cross-linking of ligands that could deteriorate the photophysical properties of QDs.

Here, we demonstrate a novel means for imparting QDs with a high thermochemical stability by successfully introducing a cross-linked block copolymer as a network ligand to QDs. Our method is based on the formation of a physical barrier on QDs, which effectively suppresses the generation of surface defects. To do this, thiol-terminated block copolymer ligands with poly(methyl methacrylate) (PMMA) and poly(glycidyl methacrylate) (PGMA), P (MMA- $b$-GMA)-SH, were carefully designed to passivate QDs. The resulting QDs are covered by a PMMA outer shell, providing high miscibility in typical optical PMMA film, and a PGMA inner shell, having epoxy groups that accomplish the cross-linking reaction via a cationic ringopening polymerization under ambient conditions without damaging the $\mathrm{QDs}^{43}$. Since no external energies (UV or heat) are applied during the cross-linking reaction, no negative effect on the photophysical properties of QDs was observed after encapsulation of the QDs. The resulting QDs are encapsulated with cross-linked polymeric protective layers that effectively passivate the $\mathrm{QD}$ surfaces. This passivation not only reduces the probability of surface oxidation but also prevents QD-thiol bonds from dissociating under harsh environmental conditions such as high temperature (either in solution or nanocomposite film states). Furthermore, we demonstrated white light-emitting diodes (WLEDs) by stacking red and green QD-PMMA nanocomposite films on blue-emitting LED chips with an easy processability and cost-effective method.

\section{Experimental section Chemicals}

Toluene, tetrahydrofuran (THF), methyl isobutyl ketone (MIBK), methanol, $n$-hexane, methyl methacrylate (MMA), glycidyl methacrylate (GMA), 2-cyano-2-propyl benzodithioate (CPBD), 2,2'-azobisbutyronitrille (AIBN), hexylamine, and tris(pentafluorophenyl)borane were all purchased from Sigma-Aldrich. The PMMA homopolymer for QD-nanocomposite films $\left(M_{n}=60,000 \mathrm{~g} / \mathrm{mol}\right.$, PDI = 1.11) was purchased from Polymer Source.

Indium acetate (In(acet) 3 , 99.995\%, metal basis), palmitic acid (PA, 99\%), 1-octadecene (ODE, 90\%, technical grade), zinc acetate $\left(\mathrm{Zn}(\text { acet })_{2}, 99.995 \%\right.$, metal basis), oleic acid (OA, 99\%), tri- $n$-octylphosphine (TOP, 99\%), Se (99.99\%, powder), S (99.998\%, powder), and trioctylamine (TOA, 99\%) were purchased from UniAm. Cadmium oxide (CdO, $\geq 99.99 \%$, metal basis), 1-dodecanethiol (DDT, $\geq 98 \%)$, zinc chloride $\left(\mathrm{ZnCl}_{2}, \geq 99.995 \%\right.$ anhydrous), and myristic acid (MA, $\geq 99 \%$ ) were purchased from Sigma-Aldrich. Tris(trimethylsilyl)phosphine $\left((\mathrm{TMS})_{3} \mathrm{P}, 99 \%\right)$ was purchased from SK chemicals. All anhydrous organic solvents were purchased from Daejung (Korea). All chemicals were used as received without further purification.

\section{Preparation of precursors}

We prepared stock precursor solutions $(0.5 \mathrm{M}$ cadmium oleate $\left[\mathrm{Cd}(\mathrm{OA})_{2}\right]$ in 1-octadecene $(\mathrm{ODE}) ; 0.5 \mathrm{M}$ zinc oleate $\left[\mathrm{Zn}(\mathrm{OA})_{2}\right]$ in $\mathrm{ODE} ; 0.5 \mathrm{M}$ indium palmitate [In $\left.(\mathrm{PA})_{3}\right] ; 2 \mathrm{M}$ selenium in tri-n-octylphosphine (TOPSe), and $2 \mathrm{M}$ sulfur in TOP (TOPS)), and each was stored under an Ar atmosphere before use.

\section{Synthesis of CdSe/ZnCdS core/shell QDs}

A detailed synthetic procedure was reported previously ${ }^{9}$. All QD syntheses were performed via the Schlenk line technique. CdO (128 mg, $1 \mathrm{mmol}$ ), MA $(685 \mathrm{mg}, 3 \mathrm{mmol})$, and ODE $(15 \mathrm{~mL})$ were mixed in a 3neck flask and heated to $240{ }^{\circ} \mathrm{C}$ under inert conditions to form the $\mathrm{Cd}(\mathrm{MA})_{2}$ complex. After the reactants turned transparent, TOPSe stock solution $(5 \mathrm{mmol}, 0.25 \mathrm{~mL}$ ) was quickly injected into the reaction flask to form a $\mathrm{CdSe}$ core $($ radius $=2 \mathrm{~nm})($ yield $=56 \%)$. After $3 \mathrm{~min}$ at $270{ }^{\circ} \mathrm{C}$, $3 \mathrm{~mL}$ of $\mathrm{Zn}(\mathrm{OA})_{2}$ stock solution $(1.5 \mathrm{mmol})$ and DDT (202 $\mathrm{mg}, 1 \mathrm{mmol}$ ) were sequentially injected to form a $0.5 \mathrm{~nm}$ thick $\mathrm{Cd}_{0.6} \mathrm{Zn}_{0.4} \mathrm{~S}$ inner shell. The reaction preceded for $30 \mathrm{~min}$, and $\mathrm{Cd}(\mathrm{OA})_{2}(1 \mathrm{mmol}, 2 \mathrm{~mL}), \mathrm{Zn}(\mathrm{OA})_{2}$ $(2 \mathrm{mmol}, 4 \mathrm{~mL})$, and TOPS $(3 \mathrm{mmol}, 1.5 \mathrm{~mL})$ were added separately into the flask within $1 \mathrm{~min}$ for the formation of a $3 \mathrm{~nm}$ thick $\mathrm{Cd}_{0.5} \mathrm{Zn}_{0.5} \mathrm{~S}$ outer shell. This reaction proceeded for $10 \mathrm{~min}$. The final products were purified several times by the precipitation/redispersion method and dispersed in toluene for further use (yield $=59 \%$ ).

\section{Synthesis of $\operatorname{InP} / \mathrm{ZnSeS}$ core/shell QDs}

$\mathrm{In}(\mathrm{PA})_{3}(0.5 \mathrm{mmol}, 1 \mathrm{~mL})$ and $\mathrm{ODE}(10 \mathrm{~mL})$ were loaded in a 3-neck flask and heated to $110^{\circ} \mathrm{C}$ under vacuum. After backfilling with Ar, the reaction flask was heated to 
$270{ }^{\circ} \mathrm{C}$. A mixed solution of (TMS) $)_{3} \mathrm{P}(62 \mathrm{mg}, 0.25 \mathrm{mmol})$ and TOP $(2.5 \mathrm{mmol}, 1.25 \mathrm{~mL})$ was rapidly injected into the reaction flask to form nuclei. The reaction temperature was maintained for $20 \mathrm{~min}$ at elevated temperature and cooled to complete the reaction. The resultant core QDs were purified via the precipitation (acetone)/redispersion (toluene) method. Final InP QDs were dispersed in toluene $(100 \mathrm{mg} / \mathrm{mL})$ and kept in a refrigerator for further reaction (yield $=45 \%$ ).

For $\mathrm{ZnSe}$ and $\mathrm{ZnS}$ shell growth, $\mathrm{Zn}(\mathrm{OA})_{2}(0.75 \mathrm{mmol}$, $1.5 \mathrm{~mL})$ and ODE $(10 \mathrm{~mL})$ were loaded in a 3-neck flask and heated to $110{ }^{\circ} \mathrm{C}$ under vacuum. After backfilling with Ar, $0.1 \mathrm{~mL}$ of InP QD solution was slowly added into the reaction flask and heated to $300^{\circ} \mathrm{C}$. At elevated temperatures, the desired amount of TOPSe precursor was injected into the reaction flask for the growth of the $\mathrm{ZnSe}$ shell. After $30 \mathrm{~min}$ of reaction, the desired amounts of $\mathrm{Zn}$ $(\mathrm{OA})_{2}$ and TOPS were injected separately into the reaction flask to grow the $\mathrm{ZnS}$ shell, and the reaction preceded for $1 \mathrm{~h}$ at $310^{\circ} \mathrm{C}$. The resultant QDs were purified twice by the precipitation/redispersion method and dispersed in toluene (yield $=52 \%$ ).

\section{Synthesis of $\mathrm{P}(\mathrm{MMA}-b-\mathrm{GMA})$-SH by RAFT polymerization}

Thiol-terminated cross-linkable polymeric ligands were synthesized via reversible addition-fragmentation chain transfer (RAFT) polymerization. First, MMA (5 g, $49.94 \mathrm{mmol})$, CPBD $(0.096 \mathrm{~g}, 0.43 \mathrm{mmol})$, and AIBN $(0.071 \mathrm{~g}, 0.043 \mathrm{mmol})$ were mixed in a Schlenk flask and degassed by argon purging. The polymerization was carried out at $75^{\circ} \mathrm{C}$ for $24 \mathrm{~h}$, and the reaction mixture was poured into cold methanol, yielding pink-colored powder of PMMA-RAFT macroinitiator. The molecular weight $\left(M_{n}\right)$ and polydispersity index (PDI) were $9,300 \mathrm{~g} / \mathrm{mol}$ and 1.23, respectively. Second, to add PGMA block, PMMARAFT macroinitiator $(3 \mathrm{~g}, \quad 0.32 \mathrm{mmol})$ was mixed with GMA $(1.6 \mathrm{~g}, 11.26 \mathrm{mmol})$ and AIBN $(0.0053 \mathrm{~g}$, $0.032 \mathrm{mmol}$ ) and polymerized at $70^{\circ} \mathrm{C}$ for $6 \mathrm{~h}$. Then, the reaction mixture was poured into cold methanol to obtain P(MMA- $b$-GMA)-RAFT block copolymer as a pink powder. The $M_{n}$ and PDI of the block copolymer were $12,100 \mathrm{~g} / \mathrm{mol}$ and 1.21 , respectively. Finally, a terminal anchoring thiol group (-SH) was prepared by nucleophilic substitution of the RAFT end group with hexylamine (four equivalents in anhydrous THF). The mixture was stirred overnight until the solution color turned pink to pale yellow, precipitated in cold methanol and dried in vacuo to produce $\mathrm{P}(\mathrm{MMA}-b-\mathrm{GMA})-\mathrm{SH}$ as a white powder.

\section{Ligand exchange}

Substitution of pristine OA ligands to polymeric ligands was performed in a single-phase reaction. Ten milligrams of QDs dissolved in $2 \mathrm{~mL}$ toluene was mixed with $40 \mathrm{mg}$ of
P(MMA- $b$-GMA)-SH dissolved in $3 \mathrm{ml}$ of toluene. The ligand exchange reaction was carried out for $48 \mathrm{~h}$ and terminated by precipitating the resulting polymer-grafted QDs (P(MMA- $b$-GMA)-QDs) with excess cold $n$-hexane. Afterward, sediments were redispersed with toluene, and precipitation-redispersion cycles were repeated three times to remove unbound polymeric or OA ligands $(1 \mathrm{mg} / \mathrm{mL}$ in MIBK).

\section{Encapsulation procedure by cross-linking of PGMA blocks}

For the formation of a cross-linked layer surrounding QDs via cationic ring-opening polymerization of the PGMA blocks, purified P(MMA- $b$-GMA)-QDs were dissolved in MIBK, and then, tris(pentafluorophenyl)borane was added (four equivalent to the total calculated amount of epoxy groups in PGMA) to initiate polymerization of epoxy groups on the PGMA blocks. The reaction flask was kept for $24 \mathrm{~h}$ to complete the cross-linking reaction. The resultant products were then precipitated with excess $n$-hexane and redispersed in toluene $(5 \mathrm{mg} / \mathrm{mL})$.

\section{Preparation of QD-PMMA nanocomposite thin films}

Glass substrates were cleaned sequentially with acetone, methanol, and isopropyl alcohol by sonication for $15 \mathrm{~min}$ before use. The composite solution was prepared by dissolving QDs and PMMA homopolymer $\left(\mathrm{M}_{\mathrm{n}}=60,000 \mathrm{~g} /\right.$ $\mathrm{mol})$ in toluene (0.5 $\mathrm{wt} \%$ and $1 \mathrm{wt} \%$, respectively). Then, thin films were fabricated by spin casting at $4000 \mathrm{rpm}$. Free-standing films for top-view TEM were prepared by floating on the surface of a buffered oxide etch (BOE) solution and transferred to TEM grids. Thin crosssectional sample (ca. $100 \mathrm{~nm}$ thick) was obtained by focused ion beam (FIB) milling.

\section{Fabrication of white QD-LED}

To a $1 \mathrm{~g}$ solution of $15 \mathrm{wt} \%$ PMMA homopolymer $\left(\mathrm{M}_{\mathrm{n}}=60,000 \mathrm{~g} / \mathrm{mol}\right)$ dissolved in chloroform, red CdSe/ $\mathrm{ZnCdS}(5 \mathrm{mg})$ and green InP/ZnSeS (20 mg) P(MMA- $b$ GMA)-QDs were added and sonicated until the solution became clear. The viscous composite solution was poured into a circular aluminum dish $(2 \mathrm{~cm}$ diameter, $0.7 \mathrm{~mm}$ depth) and dried overnight. Then, the thick QD-PMMA film was detached carefully without any damage and deposited onto a blue InGaN LED chip.

\section{Characterization}

Characterization of the polymeric ligand was performed by gel permeation chromatography (GPC) and nuclear magnetic resonance (NMR). Waters GPC with THF as the eluent was used, and calculation of $M_{n}$ was performed by calibration with linear PS standards. To monitor the ligand exchange, the hydrodynamic diameters of the QDs were measured by dynamic light scattering (DLS) using BI-200SM (Brookhaven Instruments Corporation) with a 
$616 \mathrm{~nm}$ laser. Samples were diluted with toluene at room temperature. For optical characterization, absorption and emission spectra were obtained with a V-670 UV-visible/ NIR spectrometer (Jasco Corporation) and Fluoromax-4 spectrometer (Horiba Scientific). The PL QYs of the solutions were calculated by comparing the PL QY of $\mathrm{CdS} / \mathrm{CdSe} / \mathrm{CdS}$ spherical quantum wells ${ }^{26}$ (SQWs, radius $5.1 \mathrm{~nm}$, absolute PLQY $=90 \%$ ) solution, and all measurements were carried out at the same optical densities $(\mathrm{OD}=0.1)$ dispersed in the same solvent at an excitation wavelength of $450 \mathrm{~nm}$. PLQYs were calculated by comparing the integrated area of the emission spectrum from $500 \mathrm{~nm}$ to $800 \mathrm{~nm}$ (Fig. S1). The absolute PLQY of the SQW standard in solution was measured with a QE-2100 (Otsuka Photal Electronics) at the excitation wavelength $(450 \mathrm{~nm})$. The absolute PLQY measurement systems were equipped with an integrating hemisphere, a low stray light spectrometer, and fluorescent re-excitation elimination. It should also be noted that these SQW nanocrystals exhibit extremely high stability, comparable to that of organic dyes such as rhodamine $6 \mathrm{G}$ or fluorescein, as previously demonstrated by thermal stability tests ${ }^{26}$. To confirm the reliability of the SQW standard, we measured the absolute PLQY repeatedly at various optical densities (Fig. S2). The morphologies of QDs in the PMMA matrix were examined by transmission electron microscopy (TEM) operating at $200 \mathrm{kV}$ (Tecnai 20 electron microscope). Timeresolved PL experiments were carried out by using a timecorrelated single-photon counting (TCSPC) method. The samples in solutions or films were excited by a $520 \mathrm{~nm}$ pulse (LDH-P-C-520, Picoquant). The time-resolved PL signals emitted from the samples were spectrally resolved by a monochromator and finally detected by a photomultiplier tube (PMT). The instrumental function of our time-resolved PL experimental setup is approximately $0.2 \mathrm{~ns}$. A series of EL data, including EL spectra, color rendering index (CRI), color temperature, and the Commission Internationale de l'Eclairage (CIE) color coordinates, was obtained by a diode array rapid analyzer system equipped with an integrating sphere (PSI Co. Ltd).

\section{Results and discussion}

As illustrated in Scheme 1, well-defined P(MMA- $b$ GMA)-SH ligands with $M_{n}$ values of $9.3 \mathrm{~kg} / \mathrm{mol}$ (PMMA) and $2.8 \mathrm{~kg} / \mathrm{mol}$ (PGMA) were synthesized via RAFT polymerization (Supporting Information, Fig. S3). The PMMA brush was carefully chosen owing to its good optical transparency along with its high solubility in most organic solvents that allows the practical use of corresponding nanocomposites in a range of optical films fabricated by solution processing. The PGMA block covering the periphery of the QD was designed to improve the thermal stability via the formation of crosslinked networks, given that this polymeric network structure can effectively block the formation of surface defects. The resulting $\mathrm{P}(\mathrm{MMA}-b$-GMA)-SH polymeric ligands were then mixed with the pristine QD dispersion to undergo ligand exchange. As monitored by dynamic light scattering (DLS), the hydrodynamic diameters of the $\mathrm{CdSe} / \mathrm{ZnCdS}$ (or InP/ZnSeS) QDs increase significantly from $13.5(7.8) \mathrm{nm}$ to $27.1(21.0) \mathrm{nm}$ after the ligand exchange process, indicating that the long polymeric ligands effectively replace short native oleic acid (OA) ligands in QDs (Table S1). Since the thiol end group in the ligand is tethered on the surface of the QDs, the resulting QDs are encapsulated with two distinctive layers consisting of a PGMA inner shell and PMMA outer brush (Scheme 1b).

To obtain an effective cross-linked network around QDs, at least two prerequisites need to be satisfied: high areal density (i.e., enough surface coverage) of polymer ligands on the QD surface and mild cross-linking reaction conditions to avoid unwanted side reactions on the QD surface that could affect the photophysical properties of QDs. Thermal gravitational analysis reveals that the areal chain density of polymer ligands after the ligand exchange process is 1.30 and 1.18 chains $/ \mathrm{nm}^{2}$ for CdSe and InP-based QDs, respectively (Supporting Information, Fig. S4), which is sufficient for subsequent cross-linking of the PGMA block (Supporting Information, Fig. S5). In general, the structural regime associated with the molecular picture of polymer chains grafted onto spherical particles can be described in terms of grafting density $(\rho)$ and degree of polymerization $(N)^{44,45}$. At a low grafting density of $\rho<\rho_{c}$ $\left(=R_{g}{ }^{-2}\right)$, polymers are isolated, forming mushroom-like chain conformations on the grafting surface, and such a grafting density regime is often referred to as the "mushroom regime". Only when $\rho>\rho_{c}$ do chains begin to overlap with each other and behave as brushes by which particles are effectively shielded from surface exposure. In our QD system, the grafting density $(\rho)$ is $\sim 1$ chain $/ \mathrm{nm}^{2}$, and the unperturbed radius of gyrations $\left(\mathrm{R}_{\mathrm{g}}\right)$ for PMMA with an equivalent molecular weight to $12.1 \mathrm{k}$ P(MMA-b-GMA)$\mathrm{SH}$ and that with an equivalent molecular weight to $2.8 \mathrm{k}$ PGMA-SH is estimated as $2.8 \mathrm{~nm}$ and $1.3 \mathrm{~nm}$, respectively $\left(\mathrm{R}_{\mathrm{g}, \mathrm{PMMA}}=0.025 \mathrm{M}_{\mathrm{w}}{ }^{0.5}\right)^{46}$. Provided that the conformational behavior of P(MMA-b-GMA)-SH and PGMA-SH are nearly the same as that of neat PMMA and that the perturbed radius of gyrations (by any kind of perturbations, e.g., effective excluded volume including good solvent effect in low and moderate grafting densities, many body interactions in the high grafting density regime) are larger than the unperturbed dimensions, we infer that the grafting density in our QD system $\left(\rho=1\right.$ chain $\left./ \mathrm{nm}^{2}\right)$ is not in the mushroom regime $\left(\rho<\rho_{c}\right)$ either for the entire P(MMA- $b$ GMA)-SH chain $\left(\rho_{c} \cong 0.13\right.$ chains $\left./ \mathrm{nm}^{2}\right)$ or for the grafting PGMA-SH block $\left(\rho_{c} \cong 0.6\right.$ chains $\left./ \mathrm{nm}^{2}\right)$. This suggests that there may be a sufficient number of interchain GMA 


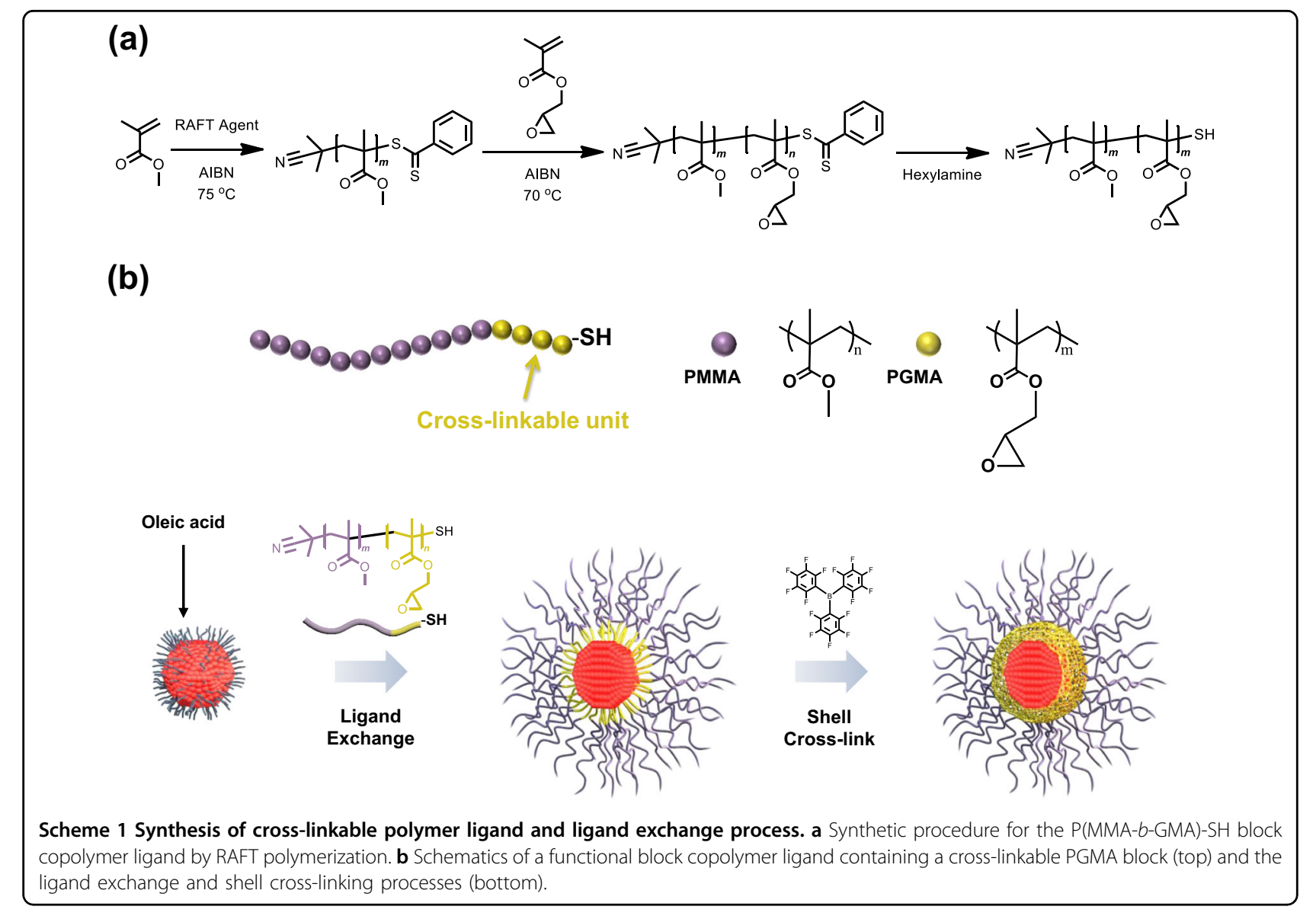

contacts that well cover the QD surface. We speculate that the high grafting density achieved even by the "grafting-to" approach in our QD system is due to the large curvature effect where the steric repulsions between grafting chains decrease more as the curvature of the particle increases. Such high grafting densities were also achieved in previous grafting-to approaches for nanoparticles with high curvatures $\left(r=1.2-1.7 \mathrm{~nm}^{-2}\right.$ for particle diameters $D=$ $2.5-10 \mathrm{~nm})^{47-50}$.

To avoid undesirable physical or chemical side effects on the QD surface, we employed the cationic ringopening polymerization of epoxides with a Lewis acid catalyst, tris(pentafluorophenyl)borane, under ambient conditions for cross-linking and corresponding encapsulation of the QDs ${ }^{51}$. As seen in Fig. 1a, b, the optical spectra of the CdSe/ZnCdS and InP/ZnSeS QDs barely change after both ligand exchange and shell cross-linking by cationic ring-opening polymerization, implying that the photophysical properties of the QDs remain intact throughout the entire QD encapsulation process. A slight decrease $(5 \%)$ in the PLQY during the ligand exchange step is attributed to the formation of hole trap sites on the QD surface during the ligand exchange process (Although the surface coverage on the QD surface would become lower after the ligand exchange process, only the marginal decrease in the PL QY is observed, suggesting that the adsorption of bulky polymeric ligands onto the QD surface does not leave unpassivated surface sites. From the ${ }^{1} \mathrm{H}-\mathrm{NMR}$ spectrum of polymer grafted QDs, it was found that $3 \mathrm{wt} \%$ of oleic acids, which corresponds to $57 \mathrm{~mol} \%$, remain even after the ligand exchange process. In this case, the chain density of residual oleic acids corresponds to 1.84 and 1.53 chains $/ \mathrm{nm}^{2}$ for CdSe and InP-based QDs, respectively. This indicates that the trap states/unpassivated surface sites are effectively passivated by both oleic acids and PMMA- $b$-PGMA-SH ligands. ${ }^{52}$. It should be highlighted that the PLQYs of QDs are highly dependent on the PGMA cross-linking method (Fig. 1c). In contrast to the mild reaction conditions for the cross-linking of PGMA using a Lewis acid catalyst (indicated with a blue arrow), the traditional cross-linking method (UV irradiation with the aid of a photoacid generator ${ }^{43}$ ) brings about a significant drop in the PLQYs of QDs via UV-induced PL quenching (indicated with a black arrow) regardless of the QD type.

To examine the thermal stabilities of QDs, QDs capped with OA, PMMA homopolymer $\left(\mathrm{M}_{\mathrm{n}}=12,000 \mathrm{~g} / \mathrm{mol}\right)$, and cross-linked P(MMA-b-GMA) (denoted as P(MMA- $b$ - 

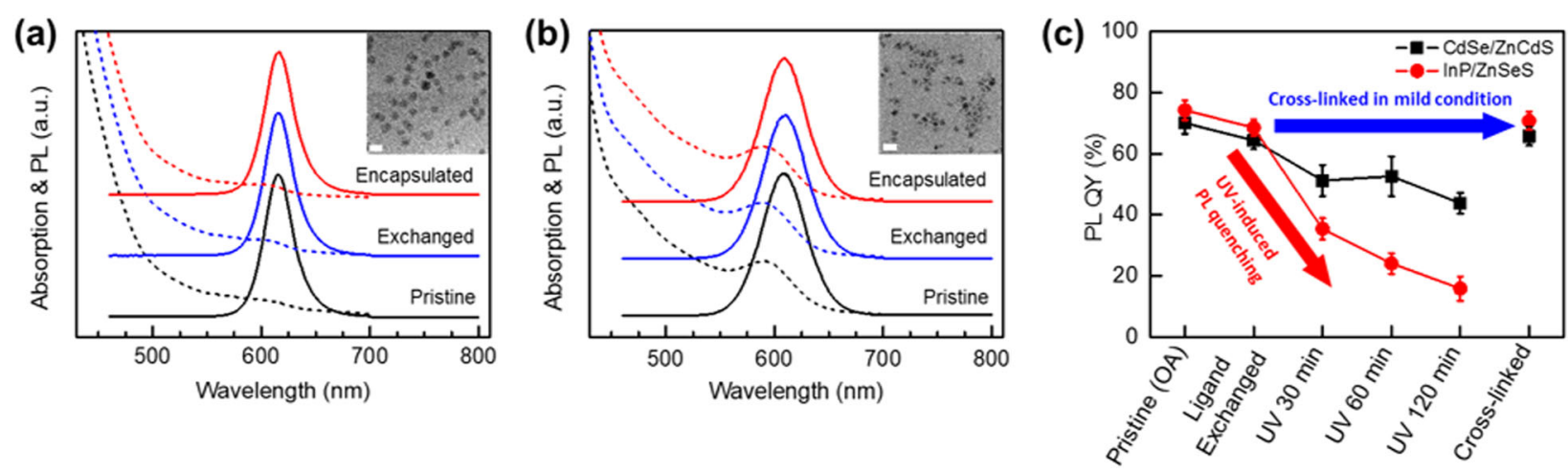

Fig. 1 Optical characteristics of CdSe/ZnCdS and InP/ZnSeS QDs during ligand exchange and cross-linking reactions. Normalized UV-vis absorbance (dashed lines) and photoluminescence (PL) spectra (bold lines) of $\mathbf{a}$ CdSe/ZnCdS QDs and $\mathbf{b} \operatorname{lnP} / \mathrm{ZnSeS}$ QDs (pristine QDs capped with OA (black, bottom), QDs tethered with P(MMA-b-GMA)-SH (blue, middle), and PGMA cross-linked P(MMA-b-XGMA)-QDs (top, red)). UV-vis and PL spectra are vertically shifted for visual clarity (insets correspond to TEM images of respective QDs. The scale bars are $20 \mathrm{~nm}$ ). c PLQY traces of CdSe/ ZnCdS QDs (black squares) and InP/ZnSeS QDs (red circles) tethered with the same P(MMA-b-GMA)-SH ligands but cross-linked with different methods. The PLQYs of QDs decrease under the UV cross-linking process (noted by black and red lines), whereas the PLQYs of QDs remain unchanged during cross-linking via cationic ring-opening polymerization of epoxides.
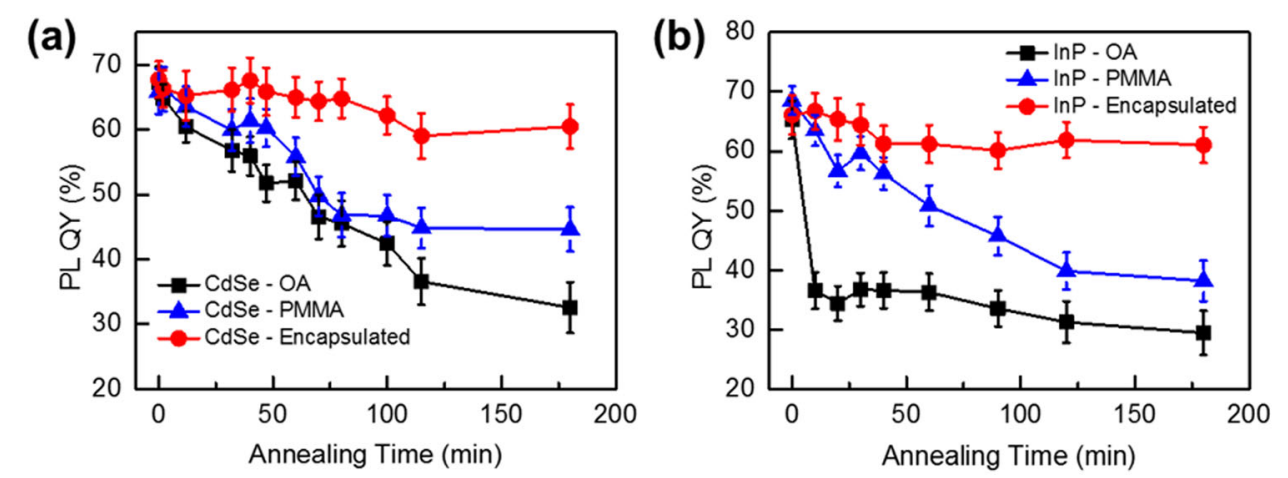

Fig. 2 PLQY of QDs as a function of thermal exposure time $\left(100^{\circ} \mathrm{C}\right.$, in toluene). a CdSe/ZnCdS QDs and $\mathbf{b} \operatorname{lnP} / \mathrm{ZnSeS}$ QDs capped with $\mathrm{OA}$ (black squares), PMMA (blue triangles), and shell-cross-linked P(MMA-b-GMA) (red circles). Error bars were obtained after at least three runs of stability tests.

XGMA)) were dispersed in toluene and annealed at $100^{\circ} \mathrm{C}$ for various times (Fig. 2). In contrast to OA- and PMMAQDs, which show a gradual PLQY decrease, P(MMA- $b$ XGMA)-QDs maintain their PLQY even at elevated temperatures, clearly reflecting that the cross-linked inner shell dramatically improves the thermal stability of QDs. Although the precise mechanism of PL quenching of QDs in solution is not fully understood, we could attribute such PL quenching to the combination of ligand dissociation and surface oxidation. Under high temperature in the presence of oxygen, sulfur atoms $\left(\mathrm{S}^{2-}\right)$ on the surface are liable to be oxidized into forms of $\mathrm{SO}_{\mathrm{x}}{ }^{-}$and leave behind uncompensated zinc atoms on the surface that serve as electron charge trap sites. The increase in the surface trap densities escalates the nonradiative recombination channels, thereby leading to the reduction of PLQY in QDs.
Ligand dissociation also presumably contributes to PL quenching following a similar mechanism, given that bonding between cation atoms on the QD surface $(\mathrm{Zn}, \mathrm{Cd})$ and thiol $\left(\mathrm{S}^{-}\right)$is unstable at elevated temperature ${ }^{53}$. In our system, the cross-linked shell structure can effectively suppress the dissociation process of thiol-terminated ligands. Furthermore, the densely cross-linked network shell acts as an insulating layer between the QD surface and outer environments and hence hinders chemical oxidation of surface atoms. From these two synergetic effects, encapsulated QDs maintain a PLQY over $85 \%$ of their initial values even after $3 \mathrm{~h}$ of annealing at elevated temperatures.

The enhanced stability of P(MMA- $b$-XGMA)-QDs against oxidation was further confirmed by direct exposure of QD solutions to ambient air or oxidative etchant 
(a)

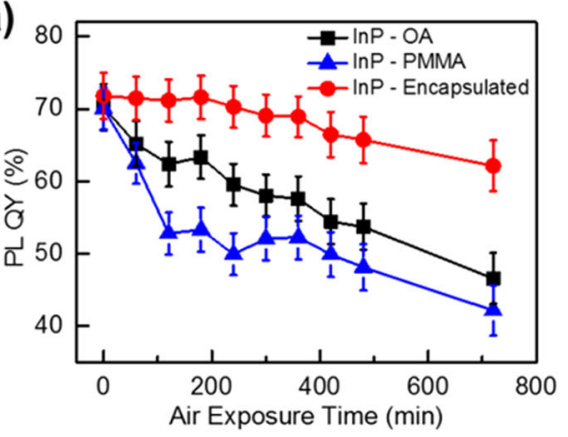

(c)

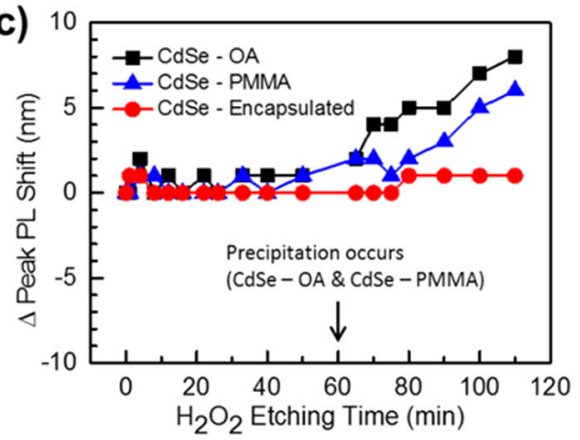

(b)

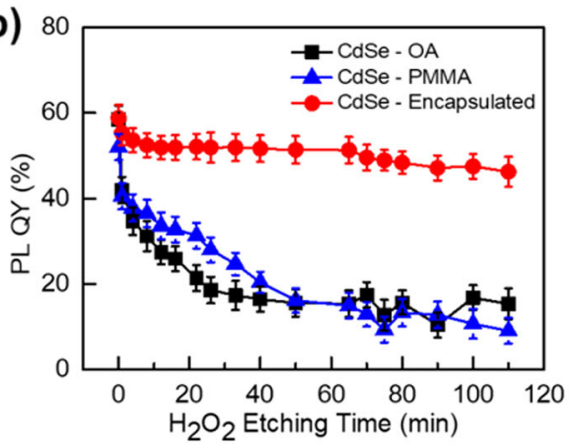

(d)

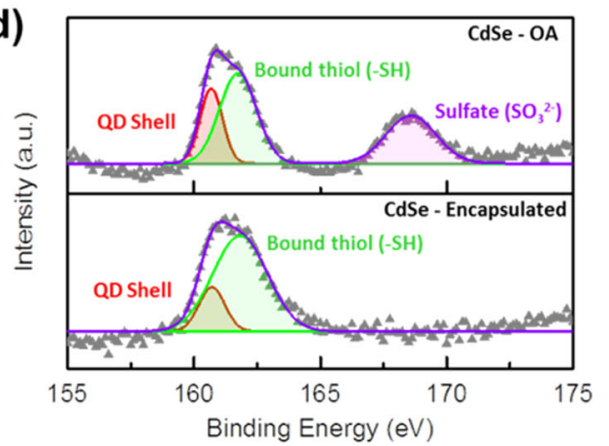

Fig. 3 Chemical and air stabilities of OA-capped QDs (black squares), PMMA-QDs (blue triangles), and P(MMA-b-XGMA)-QDs (red circles). PLQY traces of $\mathbf{a} \operatorname{lnP} / Z n S e S$ QDs upon exposure to ambient air and $\mathbf{b} \mathrm{CdSe} / \mathrm{ZnCdS}$ QDs exposed to oxidative etching conditions by the addition of $40 \mathrm{mM} \mathrm{H}_{2} \mathrm{O}_{2}$. c PL peak shift of CdSe/ZnCdS QDs upon exposure to $\mathrm{H}_{2} \mathrm{O}_{2}$. d XPS spectra of CdSe/ZnCdS QDs capped with OA ligands (top) and P (MMA-b-XGMA)-SH ligands (bottom) after exposure to $\mathrm{H}_{2} \mathrm{O}_{2}$ for $2 \mathrm{~h}$. Error bars were obtained after at least three runs of stability tests.

(i.e., hydrogen peroxide, $\mathrm{H}_{2} \mathrm{O}_{2}$ ). As seen in Fig. 3a, $\mathrm{P}$ (MMA- $b$-XGMA)-QDs exhibit greater robustness over OA- or PMMA-QDs (InP/ZnSeS QDs) under exposure to air. We attribute the decrease in the PL of the OA- and PMMA-capped QDs to reactive oxygen species in the atmosphere that undergo oxidation ${ }^{54,55}$. Although a densely cross-linked polymeric network around QDs cannot perfectly block small gaseous species such as $\mathrm{O}_{2}$, we can assume that encapsulation can reduce the probability of oxidation to some extent by screening vulnerable QD surfaces and emissive cores. In addition, since CdSe/ ZnCdS QDs possess a thicker shell $(3.5 \mathrm{~nm})$ than $\mathrm{InP} /$ ZnSeS QDs $(1.9 \mathrm{~nm})$, their stabilities against oxidation were investigated under harsher conditions by direct exposure to $\mathrm{H}_{2} \mathrm{O}_{2}$ (Fig. 3b). It should be noted that the oxidation stability test was conducted in tetrahydrofuran solution, as aqueous $\mathrm{H}_{2} \mathrm{O}_{2}$ solution is immiscible with toluene. Hence, we consider the difference in solvent refractive indices to calculate PLQYs (i.e., $\left(n_{\text {toluene }} /\right.$ $\left.n_{\text {tetrahydrofuran }}\right)^{2}$ was multiplied by the original data, where $n$ is the refractive index of the solvent). The addition of $40 \mathrm{mM} \mathrm{H}_{2} \mathrm{O}_{2}$ into the QD dispersion promptly yields a dramatic decrease in the PLQY of OA-QDs and PMMAQDs. The QDs eventually aggregate and precipitate after $\sim 1 \mathrm{~h}$ of etching. Moreover, their PL peak redshifted, as shown in Fig. 3c. In contrast, the encapsulated CdSe/
ZnCdS QDs did not exhibit any notable aggregation or redshift of the PL peak even after etching with $\mathrm{H}_{2} \mathrm{O}_{2}$, owing to the protective cross-linked shell on the QD surface. To monitor the effect of the protective shell against oxidation, we investigated the surface of the CdSe/ ZnCdS QDs by X-ray photoelectron spectroscopy (XPS) after $2 \mathrm{~h}$ of $\mathrm{H}_{2} \mathrm{O}_{2}$ etching. The $\mathrm{S} 2 \mathrm{p}$ peak in Fig. 3d shows the coexistence of the peaks from the shell of QDs $(160.7 \mathrm{eV})$ and from the bound thiol $(161.8 \mathrm{eV})$. The sulfate peak $(168.6 \mathrm{eV})$ as a result of sulfur oxidation observed in the OA-capped QDs (top) was absent in the case of shell cross-linked CdSe/ZnCdS QDs (bottom), clearly indicating that the protective shell also effectively retards oxidation processes ${ }^{56}$.

Homogeneous distribution of QDs in the matrix is a critical and basic requirement for solution-based devicefabrication processes, since massive aggregation of QDs causes poor reproducibility of the product, such as high haze and position-dependent optical quality. In addition, a close interparticle distance due to the aggregation of QDs promotes exciton transfer among QDs, which escalates the probability for photoexcited excitons to undergo nonradiative recombination processes ${ }^{57,58}$. To demonstrate the dispersion quality of the QDs in PMMA, composite films with OA- and P(MMA- $b$-XGMA)-QDs were prepared and characterized by TEM. As shown in Fig. 4a, c, OA-QDs are 



Fig. 4 Morphologies of QD-PMMA nanocomposite films. a-d Top-view and $\mathbf{e}-\mathbf{h}$ cross-sectional TEM images and $\mathbf{i}-\mathbf{l}$ AFM height profiles $(5 \times$ $5 \mu \mathrm{m})$ of QD-PMMA nanocomposite films containing CdSe/ZnCdS QDs capped by a, e, i OA and $\mathbf{b}, \mathbf{f}, \mathbf{j}$ P(MMA-b-XGMA)-SH ligands, and InP/ZnSeS QDs capped by $\mathbf{c}, \mathbf{g}, \mathbf{k}$ OA and $\mathbf{d}, \mathbf{h}, \mathbf{I}$ P(MMA-b-XGMA)-SH ligands. The scale bars in $\mathbf{a}-\mathbf{d}$ and $\mathbf{e}-\mathbf{h}$ are $200 \mathrm{~nm}$ and $100 \mathrm{~nm}$, respectively. The $S_{q}$ values in $\mathbf{i}-\mathbf{I}$ represent the root mean square height, corresponding to the surface roughness of films.

massively aggregated within the spin-cast PMMA film, mainly due to the immiscibility of PMMA and the aliphatic ligand. In contrast, the encapsulated QDs with the outer PMMA brush layer are well dispersed within the PMMA matrix without any aggregation (Fig. 4b, d). From the crosssectional TEM images, it can be clearly seen that OA-QDs are segregated into the top surface and the bottom interface of films, whereas P(MMA- $b$-XGMA)-QDs are well distributed throughout the film thickness (Fig. 4e-h). Furthermore, the surface morphologies of QDnanocomposite films were investigated by atomic force microscopy (AFM), as shown in Fig. 4i-l. We first examined the height profiles of OA-QD nanocomposite films (Fig. 4i, k), where apparent structural instabilities were clearly observed. The roughness on the PMMA film is mainly due to the aggregation of immiscible OA-QDs, possessing lower surface energy $\left(\gamma_{O A \text {-air }}=32.1 \mathrm{~mJ} / \mathrm{m}^{2}\right)$ than PMMA $\left(\gamma_{\text {PMMA-air }}=41.1 \mathrm{~mJ} / \mathrm{m}^{2}\right)^{59,60}$, into the air/ PMMA interface. However, smooth and homogeneous height profiles were obtained in the P(MMA- $b$-XGMA)QD nanocomposite films (Fig. 4j, l), suggesting that these QDs are well distributed throughout the film thickness without surface segregation/aggregation, consistent with top-view and cross-sectional TEM images.

To evaluate the thermal stability of shell cross-linked QDs in the nanocomposite films, we characterized the photophysical properties of QDs in films with time-correlated single-photon counting (TCSPC) measurements (Fig. 5). A fast decay component appears for OA-QDs after thermal annealing at $100{ }^{\circ} \mathrm{C}$ (Fig. 5a, c), representing the rise of fast nonradiative recombination processes under heat exposure. By contrast, the PL decay curves of P(MMA- $b$-XGMA)QDs barely change upon thermal annealing regardless of QD type (Fig. 5b, d), strongly suggesting that the photophysical properties of QD-nanocomposite films remain intact by cross-linked polymer shells. It is also worth noting that the rise of nonradiative recombination becomes more pronounced for InP/ZnSeS QDs (80\% PLQY loss during thermal annealing $\left(\mathrm{A} / \mathrm{A}_{0}=0.2\right)$, Fig. $\left.5 \mathrm{c}\right)$, whose shell thickness is thinner than that of $\mathrm{CdSe} / \mathrm{ZnCdS}$ QDs (40\% PLQY loss during thermal annealing $\left(\mathrm{A} / \mathrm{A}_{0}=0.6\right)$, Fig. 5a). The disparity observed in the stability test of QDs in turn suggests that the thick inorganic shell also helps to enhance the stability of QDs. Apparently, the most stable QD- 

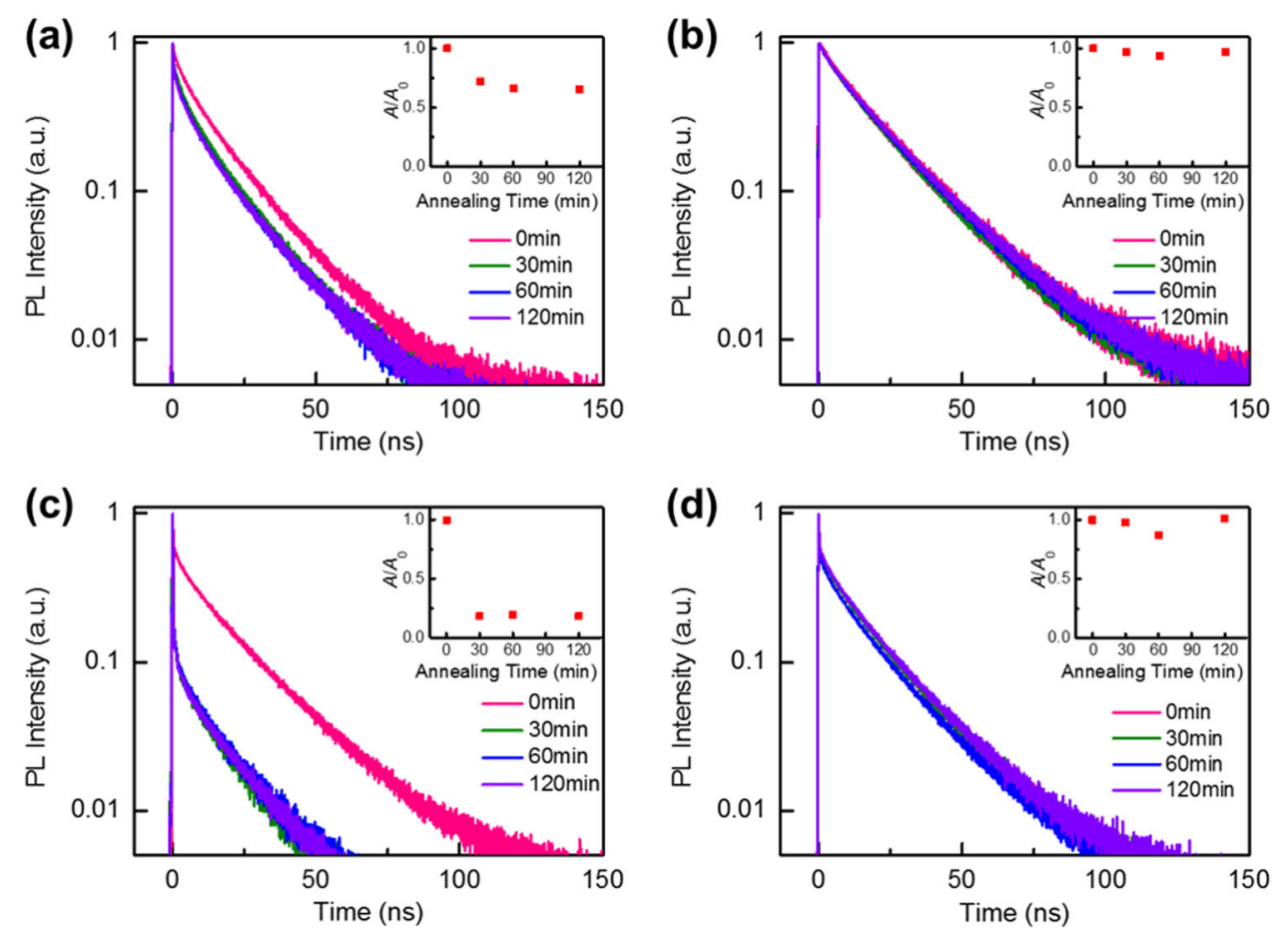

Fig. 5 Time-resolved PL spectra of QD-PMMA nanocomposite films as a function of thermal exposure time $\left(100^{\circ} \mathrm{C}\right)$. $\mathrm{PL}$ decay dynamics of $\mathrm{CdSe} / \mathrm{ZnCdS}$ QDs capped with a OA ligands and $\mathbf{b}$ P(MMA-b-XGMA)-SH ligands and InP/ZnSeS QDs capped with $\mathbf{c}$ OA ligands and $\mathbf{d}$ P(MMA-bXGMA)-SH ligands at various annealing times of the QD-nanocomposite films (insets represent the area under the time-resolved PL curves of annealed films (A) divided by that of pristine films $\left(A_{0}\right)$ as a function of annealing time).

\section{(a)}

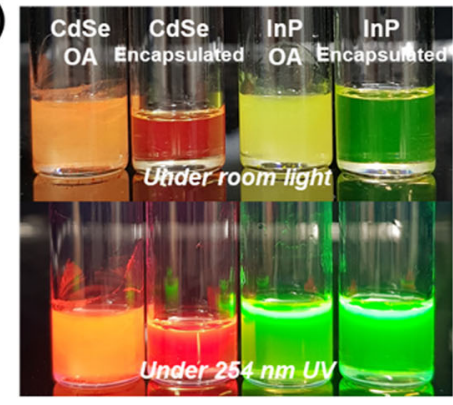

(c)

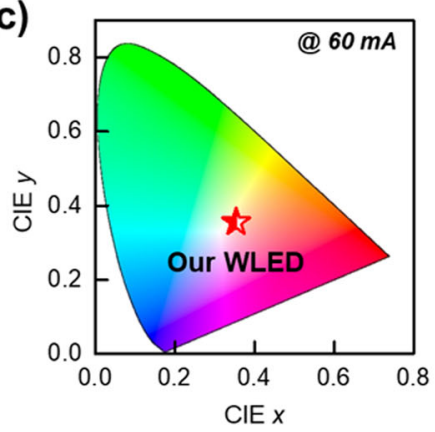

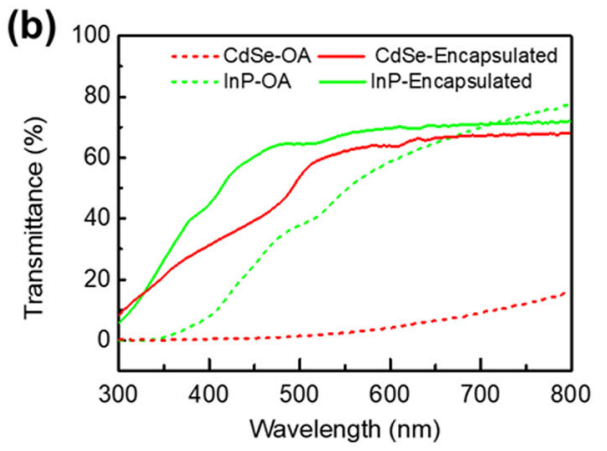

(d)

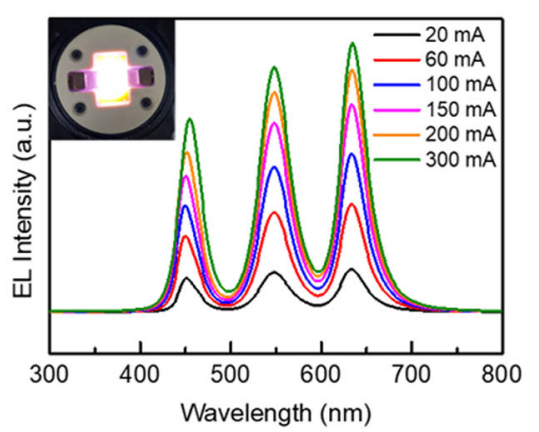

Fig. 6 EL Characteristics of downconverting white QD-LED. Photograph of a red CdSe/ZnCdS and green InP/ZnSeS QDs with OA and P(MMA-bXGMA)-SH ligands and $\mathbf{b}$ transmittance of respective thick PMMA films in the visible range. $\mathbf{c}$ CIE color coordinates and $\mathbf{d}$ EL spectra (inset: operating EL image) of the QD-based white downconversion device. 
nanocomposite film was obtained when exploiting the advantages of both the thicker inorganic shell and the passivation of QDs with cross-linked polymer ligands (Fig. 5b).

Finally, a QD-based white LED was demonstrated using a blue InGaN LED chip (with a $450 \mathrm{~nm}$ emission peak) and red and green QDs. We fabricated red and green plates by mixing PMMA and QDs and molded them into thin aluminum dishes. When films were prepared by pristine OAQDs, the composite solution was highly opaque, and the transmittance of the fabricated film was poor due to the low dispersion of QDs within the PMMA matrix. However, P(MMA- $b$-XGMA)-QDs having PMMA outer brushes were well dispersed, and films also manifested relatively high transmittance in the visible range (Fig. 6a, b). A series of EL data showed true white emission by downconverting the blue emission of the LED chip into red and green QD emission. The color coordinate was $(0.350,0.349)$ within the CIE chromaticity diagram, and the color temperature and CRI were $4833 \mathrm{~K}$ and 72.4, respectively (Fig. 6c). The EL peak contribution ratio of RGB color was maintained in the current range of 20-300 $\mathrm{mA}$, indicating the fabrication of a highly stable QD-based optical device (Fig. 6d).

\section{Conclusions}

Enhancing the stability of semiconductor QDs has been highly challenging, and many of the developed methods have intrinsic limitations or difficulties for application to final products such as optical devices. In this work, we introduced a cross-linkable polymeric ligand into QDs to form a well-defined protective shell on the QD surface. The cross-linking reaction was carried out under mild ambient conditions so that the original PLQY of QDs was not affected. Consequently, it was demonstrated that the overall stability of the shell cross-linked QDs is drastically improved and is sufficient to tolerate harsh external environments such as heat and chemical oxidation. This is a very simple yet efficient strategy to enhance the stability of QDs, which does not require any sophisticated manipulation of the intrinsic structure or geometry of QDs. Therefore, we anticipate that the present approach will pave the way for practicable use of QDs in a variety of light-emitting applications, particularly in high power light-emitting sources.

\footnotetext{
Acknowledgements

This work was supported by the National Research Foundation of Korea (NRF) grant funded by the Korean government (MSIT) (No. 2018 R1A2B2004508 and 2016M3A7B4910619) and by the Creative Materials Discovery Program through NRF grant funded by MSIT (2018M3D1A1058536).

\section{Author details}

${ }^{1}$ Department of Chemical and Biological Engineering, Korea University, Seoul 02841, Republic of Korea. ${ }^{2}$ Department of Chemical and Biomolecular Engineering, Korea Advanced Institute of Science and Technology (KAIST), Daejeon 34141, Republic of Korea. ${ }^{3}$ School of Chemical and Biological Engineering, Seoul National University, Seoul 08826, Republic of Korea. ${ }^{4}$ Department of Chemistry, Korea University, Seoul 02841, Republic of Korea.
}

${ }^{5}$ Department of Materials Science and Engineering, Hongik University, Seoul 04066, Republic of Korea. 'SKKU Advanced Institute of Nano Technology (SAINT), Sungkyunkwan University, Suwon, Gyeonggi 16419, Republic of Korea. ${ }^{7}$ Functional Composite Materials Research Center, Institute of Advanced Composites Materials, Korea Institute of Science and Technology, Wanju, Jeonbuk 55324, Republic of Korea

\section{Conflict of interest}

The authors declare that they have no conflict of interest.

\section{Publisher's note}

Springer Nature remains neutral with regard to jurisdictional claims in published maps and institutional affiliations.

Supplementary information is available for this paper at https://doi.org/ 10.1038/s41427-020-0200-4.

Received: 28 September 2019 Revised: 27 December 2019 Accepted: 7 January 2020.

Published online: 21 February 2020

\section{References}

1. Murray, C. B., Norris, D. J. \& Bawendi, M. G. Synthesis and characterization of nearly monodisperse $\mathrm{CdE}$ ( $E=$ sulfur, selenium, tellurium) semiconductor nanocrystallites. J. Am. Chem. Soc. 115, 8706-8715 (1993).

2. Murray, C. B., Kagan, C. R. \& Bawendi, M. G. Synthesis and characterization of monodisperse nanocrystals and close-packed nanocrystal assemblies. Annu. Rev. Mater. Sci. 30, 545-610 (2000).

3. Alivisatos, A. P. Semiconductor clusters, nanocrystals, and quantum dots. Science 271, 933-937 (1996).

4. Sun, Q. et al. Bright, multicolored light-emitting diodes based on quantum dots. Nat. Photonics 1, 717 (2007).

5. Coe, S., Woo, W.-K., Bawendi, M. G. \& Bulović, V. Electroluminescence from single monolayers of nanocrystals in molecular organic devices. Nature $\mathbf{4 2 0}$, 800 (2002).

6. Colvin, V., Schlamp, M. \& Alivisatos, A. P. Light-emitting diodes made from cadmium selenide nanocrystals and a semiconducting polymer. Nature $\mathbf{3 7 0}$, 354 (1994).

7. Shirasaki, Y., Supran, G. J., Bawendi, M. G. \& Bulović, V. Emergence of colloidal quantum-dot light-emitting technologies. Nat. Photonics 7, 13 (2013).

8. Kwak, J. et al. Bright and efficient full-color colloidal quantum dot lightemitting diodes using an inverted device structure. Nano Lett. 12, 2362-2366 (2012).

9. Lim, J. et al. Influence of Shell Thickness on the Performance of Light-Emitting Devices Based on CdSe/Zn1-XCdXS Core/Shell Heterostructured Quantum Dots. Adv. Mater. 26, 8034-8040 (2014).

10. Howes, P. D., Chandrawati, R. \& Stevens, M. M. Colloidal nanoparticles as advanced biological sensors. Science 346, 1247390 (2014).

11. Alivisatos, P. The use of nanocrystals in biological detection. Nat. Biotechnol. 22, 47 (2004).

12. Hwang, D. K. et al. Ultrasensitive PbS quantum-dot-sensitized InGaZnO hybrid photoinverter for near-infrared detection and imaging with high photogain. NPG Asia Mater. 8, e233 (2016).

13. Han, Y. D. et al. Quantum dot and $\pi$-conjugated molecule hybrids: nanoscale luminescence and application to photoresponsive molecular electronics. NPG Asia Mater. 6, e103 (2014).

14. Kamat, P. V. Quantum dot solar cells. The next big thing in photovoltaics. J. Phys. Chem. Lett. 4, 908-918 (2013).

15. Chen, K.J. et al. The influence of the thermal effect on CdSe/ZnS quantum dots in light-emitting diodes. J. Light. Technol. 30, 2256-2261 (2012).

16. Woo, J. Y., Kim, K. N., Jeong, S. \& Han, C.-S. Thermal behavior of a quantum dot nanocomposite as a color converting material and its application to white LED. Nanotechnology 21, 495704 (2010).

17. Zhao, $Y$. et al. High-temperature luminescence quenching of colloidal quantum dots. ACS Nano 6, 9058-9067 (2012).

18. Dabbousi, B. O. et al. CdSe) ZnS core- shell quantum dots: synthesis and characterization of a size series of highly luminescent nanocrystallites. J. Phys. Chem. B 101, 9463-9475 (1997).

19. Inerbaev, T. M. et al. Quantum chemistry of quantum dots: Effects of ligands and oxidation. J. Chem. Phys. 131, 044106 (2009). 
20. Katari, J. B., Colvin, V. L. \& Alivisatos, A. P. X-ray photoelectron spectroscopy of CdSe nanocrystals with applications to studies of the nanocrystal surface. J. Phys. Chem. 98, 4109-4117 (1994).

21. Tata, M., Banerjee, S., John, V. T., Waguespack, Y. \& McPherson, G. L. Fluorescence quenching of CdS nanocrystallites in AOT water-in-oil microemulsions. Colloids Surf. A 127, 39-46 (1997)

22. Wang, $\mathrm{N}$. et al. Highly luminescent silica-coated $\mathrm{CdS} / \mathrm{CdSe} / \mathrm{CdS}$ nanoparticles with strong chemical robustness and excellent thermal stability. Nanotechnology 28, 185603 (2017).

23. Chen, Y. et al. "Giant" Multishell CdSe Nanocrystal Quantum Dots with Suppressed Blinking. J. Am. Chem. Soc. 130, 5026-5027 (2008).

24. Fu, Y. et al. Excellent stability of thicker shell CdSe@ZnS/ZnS quantum dots. RSC Adv. 7, 40866-40872 (2017).

25. Gong, K. \& Kelley, D. F. Lattice strain limit for uniform shell deposition in zincblende CdSe/CdS quantum dots. J. Phys. Chem. Lett. 6, 1559-1562 (2015).

26. Jeong, B. G. et al. Colloidal spherical quantum wells with near-unity photoluminescence quantum yield and suppressed blinking. ACS Nano 10, 9297-9305 (2016).

27. Nann, T. \& Mulvaney, P. Single quantum dots in spherical silica particles. Angew. Chem. Int. Ed. 43, 5393-5396 (2004).

28. Jun, S., Lee, J. \& Jang, E. Highly luminescent and photostable quantum dot-silica monolith and its application to light-emitting diodes. ACS Nano $\mathbf{7}$, 1472-1477 (2013).

29. Jang, E.-P. et al. Near-complete photoluminescence retention and improved stability of $\operatorname{InP}$ quantum dots after silica embedding for their application to on-chip-packaged light-emitting diodes. RSC Adv. 8, 10057-10063 (2018).

30. Li, Z., Yao, W., Kong, L., Zhao, Y. \& Li, L. General method for the synthesis of ultrastable core/shell quantum dots by aluminum doping. J. Am. Chem. Soc. 137, 12430-12433 (2015).

31. Koole, R. et al. On the Incorporation Mechanism of Hydrophobic Quantum Dots in Silica Spheres by a Reverse Microemulsion Method. Chem. Mater. 20, 2503-2512 (2008).

32. Rogach, A. L., Nagesha, D., Ostrander, J. W., Giersig, M. \& Kotov, N. A. "Raisin Bun"-Type Composite Spheres of Silica and Semiconductor Nanocrystals. Chem. Mater. 12, 2676-2685 (2000).

33. Anderson, B. D., Wu, W.-C. \& Tracy, J. B. Silica Overcoating of CdSe/CdS Core/ Shell Quantum Dot Nanorods with Controlled Morphologies. Chem. Mater. 28, 4945-4952 (2016).

34. Gao, Y. \& Peng, X. Photogenerated Excitons in Plain Core CdSe Nanocrystals with Unity Radiative Decay in Single Channel: The Effects of Surface and Ligands. J. Am. Chem. Soc. 137, 4230-4235 (2015).

35. Bae, W. K. et al. Highly Effective Surface Passivation of PbSe Quantum Dots through Reaction with Molecular Chlorine. J. Am. Chem. Soc. 134, 20160-20168 (2012)

36. Woo, J. Y. et al. Air-Stable PbSe Nanocrystals Passivated by Phosphonic Acids. J. Am. Chem. Soc. 138, 876-883 (2016).

37. Flanagan, J. C. \& Shim, M. Enhanced Air Stability, Charge Separation, and Photocurrent in CdSe/CdTe Heterojunction Nanorods by Thiols. J. Phys. Chem. C. 119, 20162-20168 (2015).

38. Wang, A. et al. Bright, efficient, and color-stable violet ZnSe-based quantum dot light-emitting diodes. Nanoscale 7, 2951-2959 (2015).

39. Guo, W., Li, J. J., Wang, Y. A. \& Peng, X. Luminescent CdSe/CdS core/shell nanocrystals in dendron boxes: superior chemical, photochemical and thermal stability. J. Am. Chem. Soc. 125, 3901-3909 (2003).

40. Wang, Y. A., Li, J. J., Chen, H. \& Peng, X. Stabilization of inorganic nanocrystals by organic dendrons. J. Am. Chem. Soc. 124, 2293-2298 (2002).
41. Dong, $\mathrm{H}$. et al. One-pot synthesis of robust core/shell gold nanoparticles. J. Am. Chem. Soc. 130, 12852-12853 (2008).

42. Yoo, M. et al. Facile Synthesis of Thermally Stable Core- Shell Gold Nanoparticles via Photo-Cross-Linkable Polymeric Ligands. Macromolecules 43, 3570-3575 (2010).

43. Chemtob, A., Versace, D.-L., Belon, C., Croutxé-Barghorn, Cl \& Rigolet, S. Concomitant organic- inorganic UV-curing catalyzed by photoacids. Macromolecules 41, 7390-7398 (2008).

44. Ohno, K., Morinaga, T., Takeno, S., Tsujii, Y. \& Fukuda, T. Suspensions of silica particles grafted with concentrated polymer brush: Effects of graft chain length on brush layer thickness and colloidal crystallization. Macromolecules 40, 9143-9150 (2007).

45. Dukes, D. et al. Conformational transitions of spherical polymer brushes: synthesis, characterization, and theory. Macromolecules 43, 1564-1570 (2010).

46. O'Reilly, J., Teegarden, D. \& Wignall, G. Small-and intermediate-angle neutron scattering from stereoregular poly (methyl methacrylate). Macromolecules $\mathbf{1 8}$, 2747-2752 (1985).

47. Corbierre, M. K., Cameron, N. S. \& Lennox, R. B. Polymer-stabilized gold nanoparticles with high grafting densities. Langmuir 20, 2867-2873 (2004).

48. Jang, S. G., Kim, B. J., Hawker, C. J. \& Kramer, E. J. Bicontinuous block copolymer morphologies produced by interfacially active, thermally stable nanoparticles. Macromolecules 44, 9366-9373 (2011).

49. Kwon, T. et al. Size-controlled polymer-coated nanoparticles as efficient compatibilizers for polymer blends. Macromolecules 44, 9852-9862 (2011).

50. Asai, M., Zhao, D. \& Kumar, S. K. Role of grafting mechanism on the polymer coverage and self-assembly of hairy nanoparticles. ACS Nano 11, 7028-7035 (2017).

51. Perez-Baena, I. et al. Endowing single-chain polymer nanoparticles with enzyme-mimetic activity. ACS Macro Lett. 2, 775-779 (2013).

52. Jeong, S. et al. Effect of the thiol- thiolate equilibrium on the photophysical properties of aqueous CdSe/ZnS nanocrystal quantum dots. J. Am. Chem. Soc. 127, 10126-10127 (2005).

53. Bain, C. D. et al. Formation of monolayer films by the spontaneous assembly of organic thiols from solution onto gold. J. Am. Chem. Soc. 111, 321-335 (1989).

54. Cordero, S., Carson, P., Estabrook, R., Strouse, G. \& Buratto, S. Photo-activated luminescence of CdSe quantum dot monolayers. J. Phys. Chem. B 104, 12137-12142 (2000).

55. Van Sark, W. G. et al. Photooxidation and photobleaching of single CdSe/ZnS quantum dots probed by room-temperature time-resolved spectroscopy. J. Phys. Chem. B 105, 8281-8284 (2001).

56. Fantauzzi, M., Elsener, B., Atzei, D., Rigoldi, A. \& Rossi, A. Exploiting XPS for the identification of sulfides and polysulfides. RSC Adv. 5, 75953-75963 (2015).

57. Reitinger, N., Hohenau, A., Köstler, S., Krenn, J. R. \& Leitner, A. Radiationless energy transfer in CdSe/ZnS quantum dot aggregates embedded in PMMA. Phys. Status Solidi A 208, 710-714 (2011).

58. Choi, Y. J., Hwang, D., Chung, H., Kim, D. Y. \& Kim, D. Controlling the spatial distribution of quantum dots in nanofiber for light-harvesting devices. NPG Asia Mater. 7, e202 (2015).

59. Kim, J. \& Green, P. F. Phase behavior of thin film brush-coated nanoparticles/ homopolymer mixtures. Macromolecules 43, 1524-1529 (2010).

60. Meli, L., Arceo, A. \& Green, P. F. Control of the entropic interactions and phase behavior of athermal nanoparticle/homopolymer thin film mixtures. Soft Matter 5, 533-537 (2009). 